\title{
A Bayesian updating procedure for the electromechanical properties of piezoelectric energy harvesters
}

\author{
Patricio Peralta ${ }^{1, *}$, Rafael O. Ruiz ${ }^{2, * *}$, and Viviana Meruane ${ }^{1, * * *}$ \\ ${ }^{1}$ Department of Mechanical Engeneering, Universidad de Chile, Beauchef 851, Santiago, Chile \\ ${ }^{2}$ Department of Civil Engeneering, Universidad de Chile, Blanco Encalada 2002, Santiago, Chile
}

\begin{abstract}
In the last decade, several numerical and analytic procedures have been proposed to predict the dynamic behavior of piezoelectric energy harvesters (PEHs). Nevertheless, PEHs present characteristics that are difficult to control in their manufacturing process, for example the electromechanical properties of the materials present variations up to $20 \%$ of their nominal values. In that sense, the use of deterministic models to obtain accurate predictions implies to have full information about the geometry and the electromechanical properties. This work introduces a procedure to update the electromechanical properties of PEHs based on Bayesian updating techniques. The procedure requires the use of: (i) a predictive model, (ii) a prior multivariate probabilistic density function for the electromechanical properties, and (iii) experimental measurements of the harvester response. The mode of the updated electromechanical properties is identified adopting a Maximum a Posteriori estimate while the probability density function associated is obtained by applying a Laplace's asymptotic approximation. The procedure is exemplified using the experimental characterization of 20 nominally identical PEHs. Results show the capability of the procedure to update not only the electromechanical properties of each PEH but also the characteristics of the whole sample of harvesters (mandatory information for design purposes).
\end{abstract}

\section{Introduction}

The dynamic description of piezoelectric energy harvesters has been widely studied in the last decade. Different deterministic modeling techniques and simplifications have been adopted to describe their electromechanical coupling effect in order to increase the accuracy on the output power estimation. Although it is a common practice to use deterministic models to predict the input-output behavior of PEHs, perfect predictions are not expected since these devices are not exempt of uncertainties. The accuracy of the output estimation is affected mainly by three factors: (1) the mathematical model used, (2) the uncertainties on the mathematical model parameters and (3) the uncertainties related to the excitation. These uncertainties should be taken into account in order to generate robust and more plausible predictions. Nevertheless, only a limited attention has been paid in the uncertainty quantification related to model parameters in piezoelectric energy harvesters.

\footnotetext{
*e-mail: patricio.peralta@ug.uchile.cl

**e-mail: rafaelruiz@uchile.cl

***e-mail: vmeruane@ing.uchile.cl
} 
In that sense, the adequate modelling of PEH plays a determinant role for their prediction, optimization and design. Although the models available offer a good description of the physics involved in PEH, its accuracy relies in the complete knowledge of the electromechanical properties and the geometrical characteristics of the harvester. In a recent work, Ruiz and Meruane [3] observed that the manufacturers typically report these electromechanical properties with variation close to $20 \%$ of their nominal values. In that sense, the authors proposed a procedure to propagate these variations in order to identified the expected Frequency Response Function (FRF) and its corresponding confidence interval. However, the information about the uncertainties related to model parameters in PEHs on few occasions is reported by the manufacturers, which tends to overestimate the variability in the dynamic behaviour. This work introduces a procedure to update the electromechanical properties of PEHs, using measurements, based on Bayesian updating techniques.

\section{Deterministic model}

The Analytical Distributed Parameter Solution (ADPS) proposed by Erturk and Inman $[1,2]$ have been widely adopted by researchers to predict the dynamic behavior of PEHs. The approach consist on a standard modal expansion assuming an Euler-Bernoulli beam model. The model allows to identify the FRF as a function of the electromechanical and geometrical characteristic of the harvester. In that sense, the FRF of PEHs is expressed as $H(\boldsymbol{\theta}, \boldsymbol{g})$, here, $\boldsymbol{\theta}$ correspond to the electromechanical properties while $g$ are the geometrical characteristics

$$
\boldsymbol{\theta}=\left[\begin{array}{lllllll}
Y_{s} & s_{11}^{E} & d_{31} & \varepsilon_{33}^{T} & \zeta & \rho_{p} & \rho_{s}
\end{array}\right] ; \quad \boldsymbol{g}=\left[\begin{array}{llll}
L & b & h_{p} & h_{s}
\end{array}\right]
$$

The above variables corresponds to the length $L$, width $b$, thickness of the piezoelectric layer $h_{p}$, thickness of the substructure $h_{s}$, the substructures density $\rho_{s}$, the piezoelectric layers density $\rho_{p}$, the Young Modulus of the substructure $Y_{s}$, the elastic compliance at constant electric field $s_{11}^{E}$, the piezoelectric strain constant $d_{31}$, the permittivity at constant stress $\varepsilon_{33}^{T}$ and the damping ratio $\zeta$.

\section{Experimental characterization of PEHs}

The experimental identification of the FRF in PEHs requires the direct measurement of the base acceleration together to the voltage generated. As the goal of this research is to study the uncertainties in PEHs, it is required to identify the FRFs of various harvesters with the same nominal characteristics (9 bimorph PEHs are studied). The manufacturer reports the parameters indicated in Table 1.

Table 1. Nominal Characteristic of the PEHs Tested

\begin{tabular}{cl}
\hline \multicolumn{1}{c}{ Property } & \multicolumn{1}{c}{ Value } \\
\hline$\rho_{s}$ & Not Available \\
$Y_{s}$ & Not Available \\
$s_{11}^{E}$ & $16.4 \cdot 10^{-12} \mathrm{~m}^{2} \mathrm{~N}^{-1}$ \\
$d_{31}$ & $-320 \cdot 10^{-12} \mathrm{CN}^{-1}$ \\
$\varepsilon_{33}^{T}$ & $4500 \cdot\left(8.854 \cdot 10^{-12}\right) \mathrm{Fm}^{-1}$ \\
$\rho_{p}$ & $7.4 \cdot 10^{3} \mathrm{kgm}^{-3}$ \\
$L$ & $60 \mathrm{~mm}$ \\
$b$ & $10 \mathrm{~mm}$ \\
Total Thickness & $0.8 \mathrm{~mm}$ \\
\hline
\end{tabular}


The length, width and total thickness of all tested harvesters are verified with a micrometer (precision of $0.001 \mathrm{~mm}$ ). The mean and standard deviation are presented in Table 2. Here, it is possible to observe small coefficients of variation (lower than $2.6 \%$ ).

Table 2. Geometric Characteristic Measured for all PEHs Studied

\begin{tabular}{cll}
\hline Property & Mean & Coefficient of Variation \\
\hline$L$ & $60.046 \mathrm{~mm}$ & $0.1 \%$ \\
$b$ & $10.064 \mathrm{~mm}$ & $0.1 \%$ \\
Total Thickness & $0.713 \mathrm{~mm}$ & $2.6 \%$ \\
\hline
\end{tabular}

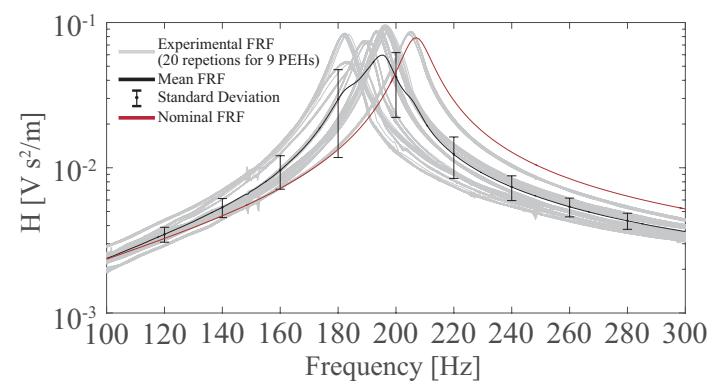

Figure 1. Measurement of the FRF of $9 \mathrm{PEH}$, all of them with identical nominal characteristics.

The FRFs of 9 PEHs are identified through 20 independent measurements for each single PEH and the results are presented in Fig. 1. Results are very interesting since the CVs observed are significant, reaching in some cases values up to $60 \%$. Furthermore, the bias between the nominal value and the measurements of the fundamental frequencies indicates that the nominal values reported by the manufacturers are not completely accurate. Additionally, it is notorious that the mean value of the thickness obtained from measurements (Table 2) differs from the nominal value reported (Table 1), differences close to $10 \%$.

\section{Probabilistic prediction using nominal values}

The procedure presented by Ruiz and Meruane [3] is employed here to quantify the expected uncertainties in the FRF. Next, a brief description of this procedure is offered. First, it is necessary to establish a deterministic model to identify the FRF of the PEH, the ADPS is used for this study. The goal is to estimate the variation of the FRF due to variations on the vector $\boldsymbol{\theta}$. In that sense, the model parameter vector $\boldsymbol{\theta}$ can be modelled as a multivariate Probabilistic Density Function (PDF) identified as $p(\boldsymbol{\theta})$. These uncertainties are propagated such that the expected value of the FRF can be estimated by solving the following probabilistic integral:

$$
E[H]=\int_{\Theta} H(\theta) p(\theta) d \theta
$$

Additionally, it is possible to identified the probability $\left(P_{o}\right)$ that $H$ exceed a certain value $H_{\text {threshold: }}$

$$
P\left(H>H_{\text {threshold }}\right)=P_{o}
$$

There are different procedures to solve Eqs. 2 and 3, being the stochastic simulation one of the most commonly procedure adopted, which is the scheme used in the present work, specifically the Monte Carlo Importance Sampling technique [3,4]. 
The probabilistic model for the model parameters $p(\boldsymbol{\theta})$ is defined by using the values reported in [3] for the geometry and variations of $\pm 20 \%$ for the electromechanical properties of the harvester (Table 1). All variables are assumed to be Gaussian.

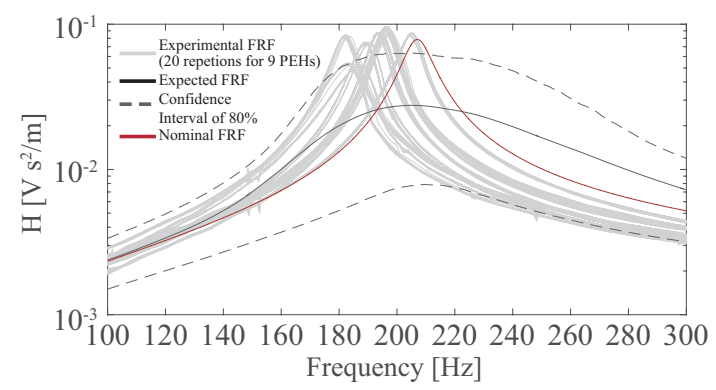

Figure 2. Comparison between a prior probabilistic prediction and measurements.

The comparison between measurements and predictions are presented in Figure 2. The interval confidence shows the capability of the numerical procedure to estimate the possible location of the actual FRF. However, the fundamental frequency estimated by the predictor is higher than the mean fundamental frequency observed. Additionally, it is observed that the dispersion used in the predictive model is considerable greater than the actual dispersion of the PEH set.

\section{Bayesian approach to update model parameters}

The following section presents a Bayesian updating method in order to identify the model parameters of a PEH. The idea behind the method is to incorporate the information gained in the experimental test to decrease the level of uncertainty associated to the main characteristics of the PEH. Based on the experimental results, it is proposed to incorporate an additive prediction error such that the estimation of the FRF can be obtained computing the following equation:

$$
h=H(\boldsymbol{\theta})+e
$$

where $e$ is an error defined by a Gaussian PDF with zero mean and a specific standard deviation $\sigma_{e}$. Under this assumption, the real system FRF $(h)$ is defined. such as:

$$
p(h \mid \boldsymbol{\theta}) \sim N\left(H(\boldsymbol{\theta}), \sigma_{e}^{2}\right)
$$

Note that Eq.5 represents the real PDF of the FRF when the model parameters $\boldsymbol{\theta}$ are known. If the information about model parameters is incomplete (model parameters defined by a PDF $p(\theta)$ ), then it is possible to propagate the uncertainties to compute the expected value of the real FRF as:

$$
E[h]=\int H(\boldsymbol{\theta}) p(\boldsymbol{\theta}) d \theta
$$

Here, $p(\boldsymbol{\theta})$ receive the name of prior PDF, since it has the initial information of the model parameters. The important aspect of the Bayesian approach is that the prior PDF $p(\boldsymbol{\theta})$ could be updated by using experimental data, then, it is obtained a posterior PDF denoted as $p(\theta \mid D)$. Where the letter $D$ stands to explicitly indicate that the PDF has been updated by the experimental data $D$. In this case, Eq.6 is re-wrote as:

$$
E[h \mid D]=\int H(\boldsymbol{\theta}) p(\boldsymbol{\theta} \mid D) d \theta
$$


The procedure to identify the posterior PDF $p(\theta \mid D)$ is presented in the following paragraphs. If the concept presented in Eq.5 is extended for multiple outputs, such that $h=$ $\left\{h_{1} h_{2} \ldots h_{M}\right\}$, then the output probability model for $M$-measurements is given by:

$$
p(h \mid \boldsymbol{\theta})=\prod_{m=1}^{M} p\left(h_{m} \mid \boldsymbol{\theta}\right)
$$

Note that Eq.8 exhibits an independence between the errors of different pairs of outputs. In other words, the prediction error at certain point does not affect the prediction error at other points. Since the experimental data $D$ is available, the Bayes Theorem can be applied here to update the probability model $p(\boldsymbol{\theta})$, such that:

$$
p(\boldsymbol{\theta} \mid D)=\frac{p(D \mid \boldsymbol{\theta}) p(\boldsymbol{\theta})}{\left.\int p(D \mid \boldsymbol{\theta}) p(\boldsymbol{\theta}) d \theta\right)}
$$

Here, $p(D \mid \boldsymbol{\theta})$ is the likelihood function which gives the probability to have the data $D$ given the model parameters $\boldsymbol{\theta}$. On the other hand, the denominator of Eq.9 is a normalizing constant which is also called the evidence of the system.

The most probable values for the model parameters can be calculated by adopting a Maximum a Posteriori estimator (MAP), where the goal is to identify the maximum of the numerator in Eq.9 (please refers to [5] for further details):

$$
\left.\boldsymbol{\theta}_{M A P}=\operatorname{argmax}[\log (D \mid \boldsymbol{\theta}) p(\boldsymbol{\theta}))\right]
$$

The Eq.9 can be solved using the Laplace method of asymptotic approximation. Wich could be used as long as the $\log$ of likelihood function be twice differentiable and significant peaked (condition attained for $M$ large) around a global maximum. The approximation behind this method relies in the assumption that the numerator of Eq.9 is a Gaussian function centered at $\boldsymbol{\theta}_{M A P}$, then, the covariance of the posterior model parameter is equal to the inverse of the Hessian matrix of $-\log (p(h \mid \boldsymbol{\theta}) p(\boldsymbol{\theta}))$ evaluated at $\boldsymbol{\theta}_{M A P}$.

The procedure is applied to the experimental measurements of $9 \mathrm{PEH}$ (Secction 4). It necessary to define first the model parameters that will be considered uncertain. The variability of the geometrical characteristics are small (lower than 2.6\%) compared with the variability associated with the electromechanical properties. In that sense, the geometrical characteristics can be considered known parameters.

The prior probability model $p(\boldsymbol{\theta})$ is defined then as an uncorrelated Gaussian distribution where the mean corresponds to the nominal values (Table 1), and CVs are equal to $20 \%$.

Table 3. Prior and Posterior PDF employed in the Bayesian procedure.

\begin{tabular}{cllll}
\hline$\theta$ & \multicolumn{1}{c}{ Prior } & \multicolumn{1}{c}{ Posterior } \\
\hline$\rho_{s}$ & $7.4 \cdot 10^{3} \mathrm{kgm}^{-3}$ & $(20 \%)$ & $4.2 \cdot 10^{3} \mathrm{kgm}^{-3}$ & $(73,1 \%)$ \\
$Y_{s}$ & $6.1 \cdot 10^{10} \mathrm{~Pa}$ & $(20 \%)$ & $3.1 \cdot 10^{9} \mathrm{~Pa}$ & $(85,3 \%)$ \\
$s_{11}^{E}$ & $16.4 \cdot 10^{-12} \mathrm{~m}^{2} / N$ & $(20 \%)$ & $20.2 \cdot 10^{-12} \mathrm{~m}^{2} / N$ & $(13.5 \%)$ \\
$d_{31}$ & $-320 \cdot 10^{-12} \mathrm{C} / \mathrm{N}$ & $(20 \%)$ & $-361.5 \cdot 10^{-12} \mathrm{C} / \mathrm{N}$ & $(8.8 \%)$ \\
$\varepsilon_{33}^{T}$ & $4500 \cdot \varepsilon_{0} \mathrm{~F} / \mathrm{m}$ & $(20 \%)$ & $4557.3 \cdot \varepsilon_{0} \mathrm{~F} / \mathrm{m}$ & $(13,6 \%)$ \\
$\rho_{p}$ & $7.4 \cdot 10^{3} \mathrm{kgm}^{-3}$ & $(20 \%)$ & $7.3 \cdot 10^{3} \mathrm{kgm}^{-3}$ & $(17.9 \%)$ \\
\hline
\end{tabular}

The results are presented in Table 3. Note that the mean values of both distributions are similar but the variances present important differences. The posterior distribution corresponds to a narrower distribution compared with the prior. This behavior is expected since the inclusion of additional information tends to decrease the uncertainties. 


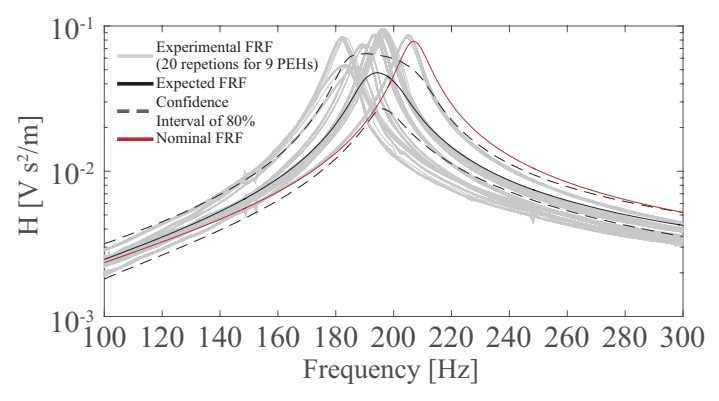

Figure 3. FRF obtained by the implementation of the Bayesian procedure

After the identification of the posterior probability density function (Table 3 ). The procedure to propagate these uncertainties is applied in order to study the new dispersion obtained in the estimation of the FRF, it is made by solving Eq.7 adopting a Monte Carlo Approach, results are presented in Fig.5, where it is observed the reduction on the confidence interval due to the updating of the model uncertainties. Additionally, it is observed a good agreement between the expected value of the FRF and the measurements obtained previously, indicating the versatility and the of this procedure to improve the prediction of the FRF in PEHs.

\section{Conclusions}

A framework to propagate uncertainties in PEH was presented. The framework presents a series of significant advantages since: (1) it is compatible with any well-known energy harvester performance predictor (deterministic models), (2) it is independent of the number of piezoelectric and substructure layers, (3) it allows to define expected values as well as confidence intervals for the FRF associated to the output voltage, and (4) it allows to update the characteristics of the PEH based on experimental data. An experimental characterization is also presented by testing 9 PEHs with identical nominal characteristics. The experimental measurements served as data to exemplified the framework presented. Results reveal the real necessity to incorporate the uncertainties in the prediction of the FRF.

\section{References}

[1] A. Erturk and D. J. Inman, J. Vib. Acoust. Trans. ASME, vol. 130, no. 4, 2008.

[2] A. Erturk and D. J. Inman, Smart Mater. Struct., vol. 18, no. 2, p. 025009, 2009.

[3] R. O. Ruiz and V. Meruane, Smart Mater. Struct., vol. 26, no. 6, p. 065003, 2017.

[4] R. D. Rosenkrantz, vol. 158. Springer Science and Business Media, 2012.

[5] J. L. Beck and A. A. Taflanidis, Int. J. Uncertain. Quantif., vol. 3, no. 4, pp. 271288, 2013. 